\title{
Optimal Detection of Certain Forms of Inappropriate Test Scores
}

\author{
Fritz Drasgow and Michael V. Levine \\ University of Illinois
}

\begin{abstract}
Optimal appropriateness indices, recently introduced by Levine and Drasgow (1984), provide the highest rates of detection of aberrant response patterns that can be obtained from item responses. In this article they are used to study three important problems in appropriateness measurement. First, the maximum detection rates of two particular forms of aberrance are determined for a long unidimensional test. These detection rates are shown to be moderately high. Second, two versions of the standardized $\ell_{0}$ appropriateness index are compared to optimal indices. At low false alarm rates, one standardized $\ell_{0}$ index has detection rates that are about $65 \%$ as large as optimal for
\end{abstract}

spuriously high (cheating) test scores. However, for the spuriously low scores expected from persons with ill-advised testing strategies or reading problems, both standardized $\ell_{0}$ indices are far from optimal. Finally, detection rates for polychotomous and dichotomous scorings of the item responses are compared. It is shown that dichotomous scoring causes serious decreases in the detectability of some aberrant response patterns. Consequently, appropriateness measurement constitutes one practical testing problem in which sig nificant gains result from the use of a polychotomous item response model.

An examinee's score on a multiple-choice test may occasionally fail to provide a representative measure of ability or achievement. Examinees' scores are spuriously high when examinees copy answers from high ability neighbors or when they have been given the answers to some questions by informants. Spuriously low scores result from alignment errors (answering the $(i+1)$ st item in the answer sheet space provided for the $i$ th answer) over a block of items, atypical education, unusually creative interpretations of normally easy items, language difficulties, suboptimal omitting strategies, and a variety of other sources.

It is important to detect aberrant response patterns. Test scores that provide unrepresentative measures of abilities can lead to misclassification errors that are expensive to test users. Furthermore, these errors can have profound implications for test takers. Individuals with spuriously high tests scores may be selected for jobs or academic programs for which they are incompetent, and spuriously low test scores may cause deserving individuals to be denied professional and academic opportunities.

Levine and Rubin (1979) used the term appropriateness measurement to denote model-based methods for detecting inappropriate test scores. Appropriateness measurement methods usually attempt to identify

APPLIED PSYCHOLOGICAL MEASUREMENT

Vol. 10, No. 1, March 1986, pp. 59-67

(C) Copyright 1986 Applied Psychological Measurement Inc.

0146-6216/86/010059-09\$1.70 
inconsistent response patterns that have correct answers to difficult items co-occurring with incorrect answers to easy items (Drasgow, 1982; Drasgow, Levine, \& Williams, 1985; Harnisch \& Tatsuoka, 1983; Levine \& Drasgow, 1982; Levine \& Rubin, 1979; Rudner, 1983; Tatsuoka, 1984). Such response patterns are expected to result from the types of test-taking anomalies described above.

\section{Previous Research}

Simulated spuriously high and spuriously low response patterns have been shown to be detectable by appropriateness measurement (Levine \& Rubin, 1979). High detection rates have been obtained despite model misspecification and item parameters estimated with error from samples that include substantial proportions of inappropriate response patterns (Levine \& Drasgow, 1982). Very high detection rates have been found when response patterns of low ability examinees have been modified to simulate cheating on $20 \%$ to $30 \%$ of the items on an 85 -item test and when response patterns of high ability examinees have been modified to simulate spuriously low responding on similar proportions of items (Drasgow et al., 1985).

The standardized $\ell_{0}$ index (Drasgow et al., 1985), denoted $z_{3}$, is easy to compute and has been found to provide relatively powerful detection of inappropriate response patterns. This index is computed from the dichotomously scored item responses, coded $u_{i}=1$ if correct and $u_{i}=0$ if incorrect, in the following way. First, the logarithm of the likelihood function at the maximum likelihood estimate $\hat{\theta}$ of $\theta$, is computed as

$\ell_{0}=\sum\left[u_{i} \log P_{i}(\hat{\theta})+\left(1-u_{i}\right) \log Q_{i}(\hat{\theta})\right]$,

where $P_{i}(\theta)$ is the probability of a correct response among examinees with ability $\theta$ and $Q_{i}(\theta)=1-$ $P_{i}(\theta)$. Then,

$z_{3}=\frac{\ell_{0}-M(\hat{\theta})}{[S(\hat{\theta})]^{1 / 2}}$,

where $M(\hat{\theta})$ and $S(\hat{\theta})$ are the conditional expected value and variance of $\ell_{0}$, respectively, given $\theta=\hat{\theta}$, that is,

$M(\hat{\theta})=\sum\left[P_{i}(\hat{\theta}) \log P_{i}(\hat{\theta})+Q_{i}(\hat{\theta}) \log Q_{i}(\hat{\theta})\right]$,

and

$S(\hat{\theta})=\sum P_{i}(\hat{\theta}) Q_{i}(\hat{\theta})\left\{\log \left[P_{i}(\hat{\theta}) / Q_{i}(\hat{\theta})\right]\right\}^{2}$.

Although the three-parameter logistic model has been used in previous research (hence " $z_{3}$ "), any of the logistic, normal ogive, or other parametric models can be used in the above expressions.

The observed rates of detection of aberrant response patterns found in earlier studies seem to be high enough for practical applications of appropriateness measurement. Nonetheless, it has not yet been determined whether an appropriateness index such as the $z_{3}$ index is powerful in an absolute sense. More specifically, none of the appropriateness indices compared to $z_{3}$ has been found to provide substantially higher rates of detection of spuriously high response patterns. Is this because no such index exists? Does a dramatically superior appropriateness index exist, but remain undiscovered? Comparative studies of available appropriateness indices (Drasgow et al., 1985; Harnisch \& Tatsuoka, 1983; Rudner, 1983) cannot answer these fundamental questions.

\section{Optimal Appropriateness Measurement}

Means for determining the statistical power of the most powerful appropriateness indices for a given form of aberrance were recently presented by Levine and Drasgow (1984). They denoted the likelihood 
of a response vector u under a model for normal response patterns, such as the three-parameter logistic, by $P_{\text {Normat }}(u)$, and the likelihood under a model for aberrant response patterns by $P_{A b e r r a n t}(u)$. The NeymanPearson Lemma can then be used to show that the highest rate of detection of aberrant response patterns is obtained by classifying un as aberrant when

$P_{\text {Aberrant }}(\mathbf{u}) \geqslant$ constant $P_{\text {Normal }}(\mathbf{u})$,

where the constant is selected to achieve a specified Type I error rate (i.e., a specified rate of misclassifying normal response patterns as aberrant).

In many cases it is easy to compute $P_{\text {Normal }}(\mathbf{u})$. For example, assume that (1) the item responses are scored dichotomously, (2) $P_{i}(t)$ is the probability of a positive response on item $i$ among normal examinees with ability $t$, (3) local independence holds, and (4) the ability density is denoted $f(t)$. Then the conditional likelihood of a given ability $t$ is

$P_{\text {Normal }}(\mathrm{a} \mid t)=\prod_{i=1}^{n} P_{i}(t)^{u_{i}}\left[1-P_{i}(t)\right]^{1-u_{i}}$,

and the unconditional likelihood is

$P_{\text {Normal }}($ ui $)=\int P_{\text {Normal }}($ ul $\mid t) f(t) d t$,

where the range of integration can be taken as the support of $f$. When $f$ and the $P_{i}$ are smooth functions, the integral in Equation 7 can be easily and accurately approximated using numerical methods.

Levine and Drasgow (1984) showed that $P_{A b e r r a n}(\mathbf{u})$ can be obtained for some forms of aberrance by taking the conditioning-integrating argument one step further. To illustrate their approach, suppose that the form of aberrance under investigation is the $10 \%$ spuriously high condition. Here it is assumed that some examinees are given the answer key to $10 \%$ of the test items which, for the statistical analysis, are taken as randomly sampled. The likelihood of al can be written conditioning on both $t$ and a particular $10 \%$ of the test items. Then, the likelihood $P_{\text {Aberrant }}(\mathbf{u} \mid t)$ is determined by averaging all the conditional probabilities obtained by selecting different aberrant items. Finally, $P_{\text {Aberrant }}(u)$ is obtained by integration as in Equation 7 or by a quadrature formula.

Direct computation of $\boldsymbol{P}_{\text {Aberrant }}(\mathrm{u} \mid t)$ for long tests would be extremely time-consuming. For example, there are about 400 billion ways of choosing $10 \%$ of the test items on an 85 -item test. Note also that to compute $\mathbb{P}_{\text {Aberrant }}(\mathrm{u}), \mathbb{P}_{\text {Aberrant }}(\mathrm{u} \mid t)$ must be evaluated for several values of $t$, so trillions of additions would be required. Of crucial import to the present research is a recursive formula presented by Levine and Drasgow (1984). The formula reduces the number of operations required to evaluate $P_{\text {Aberrant }}($ al $\mid t)$ to less than one thousand for an 85 -item test with $10 \%$ of the items spuriously high. Under these conditions, it has been found that $P_{\text {Aberrant }}$ (u) can usually be computed in less than one-tenth of a second of CDC Cyber $175 \mathrm{CPU}$ time, and costs approximately one cent at the University of Illinois.

Although optimal appropriateness indices are reasonably inexpensive to compute on a university mainframe computer, they are currently intended only as research tools. Fundamentally different expressions for $P_{A b e r r a n}(\mathbf{u})$ are obtained for spuriously high and spuriously low response patterns. Furthermore, the expression for $P_{A b e r r a n}$ (u) for, say, the $10 \%$ spuriously high condition with an 85 -item test is different from the expression for the $12 \%$ spuriously high condition, because the former conditions on the 400 billion ways of choosing $10 \%$ of the test items and the latter conditions on the 3 trillion ways of choosing $12 \%$ of the test items. Although the recursive formula can be modified to accommodate a distribution of aberrant response types, the goal at this time is to use optimal indices to answer such research questions as:

1. What is the maximum detection rate achievable by appropriateness measurement for the various benchmark types of inappropriateness?

2. How closely do selected practical, multipurpose indices approach the optimal detection rates? 
3. Can substantially better appropriateness measurement be achieved by polychotomous model indices, that is, indices that use the information in wrong answers?

\section{Three Problems in Appropriateness Measurement}

In the present research optimal indices were used to examine three questions. First, what are the upper limits on detection rates in appropriateness measurement? An answer to this question is important because it would indicate whether acceptably high detection rates are possible for important forms of aberrance on a given test. If only low rates of detection are possible for some serious forms of aberrance, then it may be prudent to lengthen the test, reduce decision makers' reliance on the test, change the testing process so that the aberrance inducing processes are inhibited (e.g., increase proctoring if copying is a problem), and so forth.

The second issue studied here concerns the virtues of the $z_{3}$ index: How powerful is the $z_{3}$ index in relation to the optimal index? If optimal indices provide only slightly higher detection rates, then $z_{3}$ would be a good candidate for use in practical settings because it is very easy to compute. In previous studies with dichotomously scored item responses $z_{3}$ was found to provide detection rates that are comparable to the rates obtained by much more sophisticated indices (e.g., Levine \& Rubin's, 1979, LR index). Nonetheless, these studies do not indicate whether $z_{3}$ provides rates of detection that are nearly optimal or whether all the indices under consideration were far from optimal.

The third problem studied concerns the loss of information about aberrance that is incurred when multiple-choice items are scored dichotomously rather than polychotomously. The polychotomous item response models currently available seem less tractable than their dichotomous counterparts. Furthermore, there are only a few examples of substantial gains from polychotomous analyses. Can substantial increases in the rates of detection of aberrant response vectors be made using a polychotomous analysis? Toward this end Drasgow et al. (1985) generalized the $z_{3}$ index to the polychotomous case. They expected this new index, labeled $z_{h}$, to be superior to $z_{3}$ because a polychotomous model provides a more fine-grained description of item responses than a dichotomous model. They found, however, that $z_{3}$ provided substantially higher rates of detection for spuriously high response patterns than did $z_{h}$. Does any index for the polychotomous data yield markedly higher rates of detection than $z_{3}$ ? Finding a polychotomous model optimal index to be clearly superior to dichotomous model indices (including the dichotomous model optimal index) would underscore the value of polychotomous item response models and the need for better polychotomous model appropriateness indices.

\section{Study 1: Dichotomous Item Responses}

\section{Method}

The problem in Study 1 was defined as: What is the upper limit on the detectability of mildly aberrant response vectors? How close to optimal is the $z_{3}$ index? Three data sets were created. The first was a normal sample of 4,000 response vectors. It was generated using three-parameter logistic item characteristic curves (ICCS) with parameters set equal to the item parameter estimates obtained from Levine and Drasgow's (1982) analysis of the 85-item April 1975 Scholastic Aptitude Test-Verbal section (SAT-v). The ability parameters were randomly sampled from a normal $(0,1)$ distribution truncated to the $[-2.05$, 2.05] interval. (The ability distribution was truncated so that the results of Study 1 would be more comparable to the results of Study 2.)

The second sample contained 2,000 response vectors subjected to the $10 \%$ spuriously high treatment. It was generated by first creating 2,000 normal response vectors by the method described above. Then 
nine items were randomly selected without replacement from each response vector and rescored as correct. The third sample also contained 2,000 response vectors. They were initially created as were the normal response vectors and then they were subjected to the $10 \%$ spuriously low treatment. Here nine items were randomly selected without replacement from each response vector and then each of these items was independently rescored as correct with probability .2 and as incorrect with probability .8 .

The three-parameter logistic maximum likelihood estimate of ability was obtained for each response vector. The simulation item parameters were taken to be known quantities. (Levine \& Drasgow, 1982, showed that values of $\ell_{0}$ computed from estimated item parameters were almost identical to values computed from the actual item parameters for a long unidimensional test. For the optimal index to be truly optimal, it must be computed using the actual item parameters.) Then, the $z_{3}$ index was computed for the response vectors in all three samples. The optimal index for the $10 \%$ spuriously high condition was computed for each response vector in the normal sample and the spuriously high sample by the algorithm described by Levine and Drasgow (1984). The $10 \%$ spuriously low optimal index was similarly computed for the spuriously low sample and the normal sample.

\section{Results}

Detection rates for various false alarm rates (Type I error rates) for the 2,000 response vectors subjected to the $10 \%$ spuriously high treatment are shown in Table 1 . It is clear that the $z_{3}$ index is close to optimal: Detection rates for $z_{3}$ are about $90 \%$ as large as the detection rates for the optimal index at corresponding false alarm rates.

Table 1 also shows that reasonably high detection rates were obtained with the optimal index. For example, $24 \%$ of the spuriously high response patterns were detected when the false alarm rate was $5 \%$. These results must be evaluated in light of the fact that, on the average, the $10 \%$ spuriously high treatment changed about four incorrect responses to correct. Consequently, the signal in this context ( 4 altered item responses) is small relative to the noise (81 item responses not altered).

The results for the 2,000 response vectors subjected to the $10 \%$ spuriously low treatment are also shown in Table 1. The detection rates for the 10\% spuriously low optimal index are somewhat lower than the rates for the $10 \%$ spuriously high optimal index. Detection rates for $z_{3}$ show larger decreases from the spuriously high condition to the spuriously low condition. Consequently, detection rates for $z_{3}$ are not very close to optimal; at low false alarm rates $z_{3}$ detects only about $65 \%$ as many spuriously low response vectors as the optimal index.

\section{Study 2: Polychatomous Item Responses}

\section{Method}

The problem in Study 2 was defined as: Is the power to detect aberrant response patterns substantially diminished when multiple-choice items are scored dichotomously? The polychotomous responses (fiveoption multiple-choice items with omitting allowed) generated for this study were created from the histograms constructed by Levine and Drasgow (1983). They used the three-parameter logistic model to estimate abilities for 49,470 examinees from the April 1975 administration of the SAT-V, formed 25 ability groups by using the 4 th, 8 th , . ., 96th percentile points from the normal distribution, and then determined the frequency of option selection (treating skipped and not reached items as a single response category) for each ability group. Probabilities of option responses were then computed by linear interpolations between ability category medians (i.e., the 2 nd, 6 th, . ., 98th percentile points from the normal distribution). Notice that response probabilities computed from these histograms for the correct options are strongly related to three-parameter logistic probabilities of correct responses. 
Table 1

Detection Rates for Response Vectors Generated by the

Three-Parameter Logistic Model for the 10\% Spuriously

High and $10 \%$ Spuriously Low Treatments

\begin{tabular}{|c|c|c|c|}
\hline $\begin{array}{l}\text { Prop. of Normals } \\
\text { Misclassified }\end{array}$ & $\frac{\text { Proportion Det }}{\text { Optimal Index }}$ & $\frac{d b y}{z_{3}}$ & $\begin{array}{c}\text { Ratio of } \\
\text { Detection Rates }\end{array}$ \\
\hline $\begin{array}{l}10 \% \text { Spuriously High } \\
.01 \\
.02 \\
.03 \\
.04 \\
.05 \\
.06 \\
.08 \\
.10 \\
.15 \\
.20 \\
.25\end{array}$ & $\begin{array}{l}.090 \\
.144 \\
.175 \\
.199 \\
.242 \\
.258 \\
.302 \\
.342 \\
.446 \\
.530 \\
.599\end{array}$ & $\begin{array}{l}.087 \\
.120 \\
.148 \\
.179 \\
.202 \\
.220 \\
.278 \\
.308 \\
.388 \\
.453 \\
.510\end{array}$ & $\begin{array}{l}.97 \\
.83 \\
.85 \\
.90 \\
.83 \\
.85 \\
.92 \\
.90 \\
.87 \\
.86 \\
.85\end{array}$ \\
\hline $\begin{array}{c}10 \% \text { Spuriously Low } \\
.01 \\
.02 \\
.03 \\
.04 \\
.05 \\
.06 \\
.08 \\
.10 \\
.15 \\
.20 \\
.25\end{array}$ & $\begin{array}{l}.076 \\
.100 \\
.130 \\
.150 \\
.170 \\
.181 \\
.210 \\
.234 \\
.312 \\
.376 \\
.444\end{array}$ & $\begin{array}{l}.046 \\
.065 \\
.079 \\
.097 \\
.114 \\
.126 \\
.160 \\
.187 \\
.250 \\
.314 \\
.372\end{array}$ & $\begin{array}{l}.61 \\
.65 \\
.61 \\
.64 \\
.67 \\
.70 \\
.76 \\
.80 \\
.80 \\
.83 \\
.84\end{array}$ \\
\hline
\end{tabular}

Note: Item responses were generated for 2,000 simulated spuriously high examinees, 2,000 simulated spuriously low examinees, and 4,000 simulated normal examinees.

Three data sets were generated in Study 2: 4,000 normal response patterns, 2,000 normal response patterns subjected to the $10 \%$ spuriously high treatment, and 2,000 normal response patterns subjected to the $10 \%$ spuriously low treatment. Abilities were randomly sampled from the normal $(0,1)$ distribution truncated to the $[-2.05,2.05]$ interval (because it was not possible to interpolate below the 2 nd percentile or above the 98th percentile). Finally, the spuriously high treatment was performed exactly as in Study 1. In the spuriously low treatment, each of the five options was selected with probability .2. Omitted responses were never modified in the spuriously low treatment nor counted toward the total of nine items.

The polychotomous generalization $z_{h}$ of $z_{3}$ was then computed for each response vector. The requisite quantities for $z_{h}$ are

$\ell_{h}=\sum_{i=1}^{n} \sum_{j=1}^{6} \delta_{j}\left(v_{i}\right) \log P_{i j}(\hat{\theta})$ 
$M_{h}(\hat{\theta})=\sum_{i=1}^{n} \sum_{j=1}^{6} P_{i j}(\hat{\theta}) \log P_{i j}(\hat{\theta})$

and

$S_{h}(\hat{\theta})=\sum_{i=1}^{n}\left\{\sum_{j=1}^{6} \sum_{k=1}^{6} P_{i j}(\hat{\theta}) P_{i k}(\hat{\theta}) \log P_{i j}(\hat{\theta}) \log \left[P_{i j}(\hat{\theta}) / P_{i k}(\hat{\theta})\right]\right\}$,

where $\quad v_{i}$ is the response to the $i$ th item, coded $v_{i}=1$ for option $\mathrm{A}, \ldots, v_{i}=5$ for option $E$, and $v_{i}=6$ for omit,

$P_{i j}(\hat{\theta})$ is the histogram probability of $v_{i}=j$ at ability $\hat{\theta}$, and

$\delta_{j}\left(v_{i}\right)=1$ if $v_{i}=j$ and 0 otherwise.

Since the histogram model likelihood function does not have a continuous first derivative and is therefore difficult to maximize, three-parameter logistic ability estimates (with the restriction $-2.05 \leqslant \hat{\theta} \leqslant 2.05$ ) were used in the above equations. The polychotomous model optimal index for the $10 \%$ spuriously high treatment was computed for the normal and spuriously high samples and the optimal index for the $10 \%$ spuriously low treatment was computed for the normal and spuriously low samples.

In many applications multiple-choice item responses are scored dichotomously. To determine the effects on appropriateness measurement of this simplification of the data, the item responses were scored dichotomously, and then $z_{3}$ and the three-parameter logistic optimal index were computed. Note that the three-parameter logistic optimal indices are not truly optimal in these analyses because the model used to analyze the data (the three-parameter logistic model) is not identical to the model used to create the data (the histogram model).

\section{Results}

Table 2 presents the results for the $10 \%$ spuriously high condition. This table shows why the detection rates of spuriously high response vectors by $z_{3}$ were found to be much higher than the rates for $z_{h}$ in the Drasgow et al. (1985) study: $z_{h}$ is a very ineffectual index for detecting spuriously high response patterns. At low false alarm rates, the $z_{h}$ index yields detection rates only about one-fourth to one-third as high as the rates for the polychotomous model optimal index. In contrast, the detection rates for $z_{3}$ are about $65 \%$ of optimal.

Table 2 also shows that the three-parameter logistic model optimal index yielded detection rates that were roughly $75 \%$ as large as the rates for the truly optimal polychotomous model index. Thus, polychotomous scoring of simulated multiple-choice item responses allows moderately higher rates of detection of spuriously high response vectors.

Table 2 also presents the results for the $10 \%$ spuriously low condition. For this treatment the polychotomous model optimal index detects almost $41 \%$ of the aberrant response vectors at a $5 \%$ false alarm rate. This high detection rate is obtained because the spuriously low modification changed more responses here than in the dichotomous case. In particular, the spuriously low treatment can change an incorrect response that is appropriate (i.e., the favorite distractor at the examinee's ability $t$ ) to an incorrect response that is inappropriate (i.e., a distractor rarely selected by examinees with abilities near $t$ ).

Table 2 shows that dichotomous scoring seriously reduces the detectability of spuriously low response vectors: The three-parameter logistic optimal index has detection rates substantially lower than the polychotomous model optimal index. At a $1 \%$ false alarm rate, the three-parameter logistic optimal index detects less than half as many aberrant response vectors as does the polychotomous model optimal index.

It is also evident in Table 2 that neither the $z_{3}$ nor the $z_{h}$ index is particularly effective. Both of these 
Table 2

Detection Rates for Response Vectors Generated by the Histogram Model for the 10\% Spuriously High and 10\% Spuriously Low Treatments

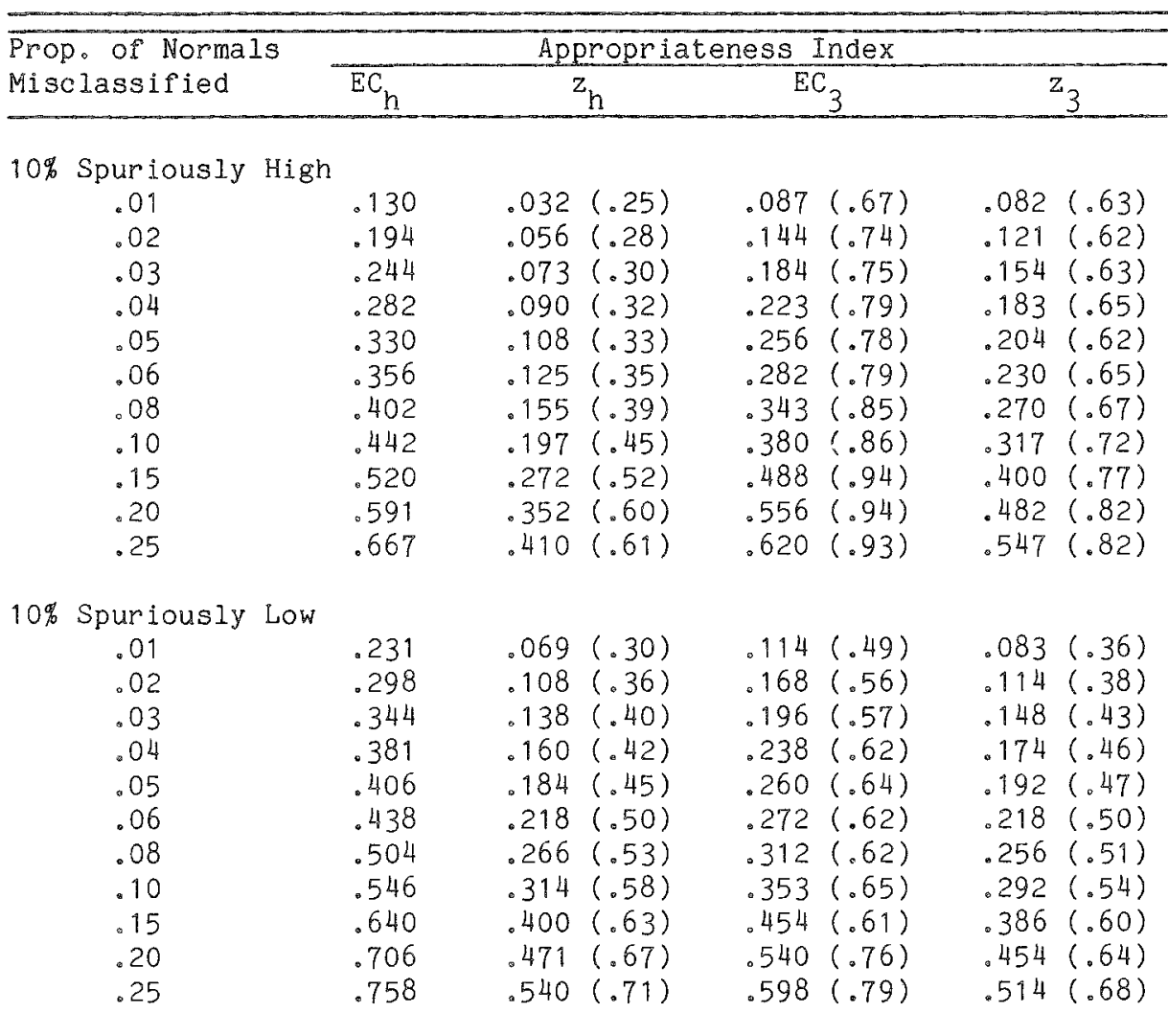

Note: Item responses were generated for 2,000 simulated spuriously high examinees, 2,000 simulated spuriously low examinees, and 4,000 simulated normal examinees. $\mathrm{EC}_{\mathrm{h}}=$ optimal histogram appropriateness index; $\mathrm{EC}_{3}=$ optimal three-parameter logistic appropriateness index. Each number in parentheses is the ratio of a nonoptimal index is hit rate and the optimal index's hit rate.

indices have detection rates that are only about $40 \%$ as large as the optimal index at low false alarm rates. Note, however, that $z_{3}$ is relatively effective in relation to the dichotomous model optimal index.

\section{Discanssion}

In the studies described above forms of aberrance that have been difficult to detect with non-optimal appropriateness indices were deliberately chosen (see Drasgow et al., 1985; Levine \& Rubin, 1979). If forms of aberrance that were easy to detect had been used, such as the $30 \%$ spuriously high and low treatments, there would have been little improvement possible. The forms of aberrance studied here certainly allowed for improvement on the detection rates of the $z_{3}$ and $z_{h}$ indices. 
The results indicate that optimal indices perform from slightly to dramatically better than previously studied indices. The least improvement was found for truly dichotomous response vectors subjected to the spuriously high manipulation. In this condition the $z_{3}$ index yielded detection rates roughly $85 \%$ to $90 \%$ as large as possible. It seems reasonable to conclude that the $z_{3}$ index is satisfactory for this situation. The $z_{3}$ index is less satisfactory for detecting response vectors that were generated by the three-parameter logistic model and then subjected to the spuriously low treatment. At low false alarm rates, $z_{3}$ detects about $65 \%$ as many aberrant response vectors as detected by the optimal index.

When item responses were created to simulate a multiple-choice test (in particular, the SAT-V), optimal appropriateness measurement using the polychotomous form of the items provides substantially improved rates of detection. The most striking results were obtained in the spuriously low condition in which both the $z_{3}$ and $z_{h}$ indices were clearly unsatisfactory. There are two implications of this result. First, it clearly shows that appropriateness measurement is one practical testing problem in which substantial gains can be made through the use of polychotomous item response models. The second implication of this research is that further work is needed on practical appropriateness indices based on polychotomous models. It is clear that $z_{h}$ is less than satisfactory for detecting spuriously high response patterns on multiple-choice tests.

In summary, the results show that moderately high detection rates can be obtained for mildly aberrant response patterns. The rates of detection are higher for polychotomous item responses than for dichotomous item responses. The $z_{3}$ index is close to optimal for dichotomous item responses generated from threeparameter logistic ICCS and subjected to the spuriously high treatment, but it is less satisfactory when dichotomous item responses are subjected to the spuriously low treatment. Finally, detection rates were substantially lower when simulated multiple-choice items were analyzed dichotomously. These results emphasize the importance of developing powerful, practical polychotomous model indices.

\section{References}

Drasgow, F. (1982). Choice of test model for appropriateness measurement. Applied Psychological Measurement, 6, 297-308.

Drasgow, F., Levine, M. V., \& Williams, E. A. (1985). Appropriateness measurement with polychotomous item response models and standardized indices. British Journal of Mathematical and Statistical Psychology, $38,67-86$.

Harnisch, D. L., \& Tatsuoka, K. K. (1983). A comparison of appropriateness indices based on item response theory. In R. Hambleton (Ed.), Applications of item response theory (pp. 104-122). Vancouver: Educational Research Institute of British Columbia.

Levine, M. V. (1985). Multilinear formula scoring: Estimation of option characteristic curves. Manuscript in preparation.

Levine, M. V., \& Drasgow, F. (1982). Appropriateness measurement: Review, critique, and validating studies. British Joumal of Mathematical and Statistical Psychology, 35, 42-56.

Levine, M. V., \& Drasgow, F. (1983). The relation between incorrect option choice and estimated ability. Educational and Psychological Measurement, 43, 675685.

Levine, M. V., \& Drasgow, F. (1984). Performance envelopes and optimal appropriateness measurement (Measurement Series 84-5). Champaign IL: University of Illinois, Department of Educational Psychology, Model-Based Measurement Laboratory.

Levine, M. V., \& Rubin, D. B. (1979). Measuring the appropriateness of multiple-choice test scores. Journal of Educational Statistics, 4, 269-289.

Rudner, L. M. (1983). Individual assessment accuracy. Journal of Educational Measurement, 20, 207-219.

Tatsuoka, $\mathbb{K}$. K. (1984). Caution indices based on item response theory. Psychometrika, 49, 95-110.

\section{Aclirnowledgments}

This research was supported by contracts N00014-79C0752, NR 154-445, and N00014-83K-0397, NR 150 518, from the Office of Naval Research, Michael V. Levine, Principal Investigator.

\section{Author's Address}

Send requests for reprints or further information to Fritz Drasgow, Department of Psychology, University of I1linois, 603 E. Daniel Street, Champaign IL. 61820, U.S.A. 